turell nicht sehr spezialisiert sind, kann beim Menschen und bei anderen Wirbeltierorganismen das essentielle Vitamin $B_{12}$-Bedürfnis nur mit einer kleinen Zahl von Vitamin $B_{12}$-Analoga befriedigt werden. Da die Vitamin $B_{12}$-Resorption beim Wirbeltierorganismus hochgradig strukturspezifisch ist und die Darmbakterien vorwiegend für den Wirtsorganismus nicht verwertbare Vitamin $\mathrm{B}_{12}$-Faktoren produzieren, außerdem die vom Intrinsic Factor abhängige Vitamin $\mathrm{B}_{12}$-Resorption im Dünndarm stattfindet im Gegensatz zu der im Caecum lokalisierten bakteriellen Biosynthese, kann der Vitamin $B_{12}$-Bedarf des Menschen und anderer Wirbeltierorganismen wohl kaum auf dem Wege einer endogenen Vitamin $B_{12}$ Synthese durch die Darmflora gedeckt werden.

Wenn auch nach den vorliegenden Ergebnissen die Nichtverwertbarkeit der endogen gebildeten Vitamin $\mathrm{B}_{12}$-Strukturen für die Deckung des Vitamin
$B_{12}$-Bedarfes des Menschen und des Säugetierorganismus geklärt sein dürfte, so ist die Bedeutung ihres Vorkommens im Darm jedoch noch weitgehend ungeklärt. Es wird der Gegenstand zukünftiger Forschungen sein, festzustellen, inwieweit den vom Wirtsorganismus nicht resorbierbaren, intestinal biosynthetisierten Vitamin $B_{12}$-Analoga eine Bedeutung als essentielle und regulativ auf die übrige, den Vitaminhaushalt des Wirtes beeinflussende Darmflora wirkende Biotika zukommt.

Den Herren Dr. Ch. Rosenblum und Dr. H. Molitor (Forschungslaboratorien Merck \& Co., Rahway/N.J., USA) verdanken wir die freundliche Überlassung biosynthetisch markierter Vitamin $B_{12}$-Analoga und den Herren Prof. K. Bernhauer und Dr. W. Friedrich (Aschaffenburger Zellstoffwerke, Stockstadt/Main) die chemische Umwandlung einiger Vitamin $\mathrm{B}_{12}$-Analoga. Der Deuts chen Forschungsgemeins chaft danken wir für die gewährte Sachbeihilfe.

\title{
Chemische und elektronenoptische Untersuchungen über die Reaktion von Chrom (III)-komplexen mit Kollagen
}

\author{
Von K. Kühn und Elisabeth Gebhardt \\ Aus dem Eduard-Zintl Institut der Technischen Hochschule Darmstadt \\ (Z. Naturforschg. 15 b, 23-30 [1960]; eingegangen am 20. November 1959)
}

\begin{abstract}
Kollagenfibrillen zeigen nach Behandeln mit gerbenden Chrom(III) komplexen im Elektronenmikroskop ein unterteiltes Querstreifungsmuster.

Es wird gezeigt, daß dabei die Ausbildung der Querstreifen auf eine ordnende Quervernetzung der Protofibrillen über die Seitenketten der sauren Aminosäuren zurückzuführen ist.
\end{abstract}

Kollagenfibrillen zeigen sowohl nach Behandeln mit Phosphorwolframsäure (PWS) als auch nach Behandeln mit gerbend wirkenden Chrom(III) -salzlösungen im Elektronenmikroskop eine hochunterteilte Querstreifung ${ }^{1}$. Die relativen Lagen der einzelnen Querstreifen längs der Periode sind nach PWS- und nach Chrombehandlung gleich ${ }^{2}$, obwohl die PWS eine Polysäure ist, während die für die Gerbung bevorzugten Sulfatochrom(III)-komplexe Polybasen sind und obwohl bei PWS-Behandlung über zehnmal mehr Masse eingelagert wird als bei Behandlung mit Chrom(III) -salzlösungen ${ }^{1,3}$.

Nach Untersuchungen von KüHn, Grassmann und HofmanN $^{1}$ über die Bindung der PWS an Kollagen

1. K. Kühn, W. Grassmann u. U. Hofmann, Z. Naturforschg. 13 b, 154 [1958].

2 K. Kühn, U. Hofmann u. W. Grassmann, Naturwissenschaften 45, 521 [1958].

3 K. KüHN, Leder 9, 217 [1958]. ist die Ausbildung einer unterteilten Querstreifung nicht so sehr ein Masseeffekt, durch die Einlagerung von Kontrastmitteln in die Dunkelteile verursacht, sondern mehr ein Ordnungseffekt.

Die Grundeinheiten oder die dünnsten Fibrilleneinheiten des Kollagens bestehen nach den Vorstellungen von RICH und $\mathrm{CRICK}^{4}$ sowie Cowan und McGavin $^{5}$ aus drei zusammengewundenen Polypeptidspiralen, die durch Wasserstoffbrücken-Bindungen miteinander verbunden sind und deren Aminosäure-Seitenketten nach außen ragen. Diese dünnsten Fasereinheiten des Kollagens werden nach Schmitr „Protofibrillen“ genannt ${ }^{6}$. Die Aminosäuren sind längs der Peptidketten der Protofibrillen so

4 A. Rich u. F. H. C. Crick, Nature [London] 176, 915 [1955].

5 P. M. Cowan u. S. McGavin, Nature [London] 176, 501 [1955].

${ }^{6}$ F. O. Schmitt, C. E. Hall u. M. A. JaKus, J. cellular comp. Physiol. 20, 11 [1942]. 
angeordnet, daß im charakteristischen Rhythmus Anhäufungen von leichten und Anhäufungen von schweren und polaren Aminosäuren aufeinander folgen $2,7,8,9$.

Bei unseren Versuchen reagieren die Protofibrillen als ungeteiltes Ganzes und werden durch die salzartige Bindung der PWS an die Guanidogruppen des Arginins so quervernetzt und geordnet, daß die Anhäufungen der schweren und polaren Aminosäuren nebeneinander zu liegen kommen und in Register gebracht werden. Es ist wahrscheinlich, daß die Querstreifung nach Chrombehandlung auf einem ähnlichen Wege zustande kommt. Vermutlich geschieht hier die ordnende Quervernetzung über die Carboxylgruppen der sauren Aminosäuren.

Die Reaktion zwischen Chrom(III)-komplexen und Kollagen ist für die Chromlederherstellung von Bedeutung. Als Maßstab für eine gute Durchgerbung des chrombehandelten Kollagens gilt die Erhöhung des Schrumpfungspunktes. Unbehandelte Fibrillen schrumpfen bei $66^{\circ}$, mit Chrom behandelte zwischen 90 und $100^{\circ}$. Diesen Effekt führt man ebenfalls auf eine Quervernetzung der Polypeptidketten über die sauren Aminosäuren zurück, deren Carboxylgruppen koordinativ in die Chromkomplexe mit einbezogen werden.

Zur Klärung dieser Zusammenhänge haben wir die Bindung kationischer und anionischer Chrom(III)-komplexe an Kollagen, ihre Gerbwirkung und die dabei entstehende Querstreifung untersucht. Wir hofften so, unsere Vorstellungen über die Entstehung der unterteilten Querstreifung zu bestätigen und etwas tiefer in die Theorie der Chromgerbung einzudringen.

Besonders wichtig war die Frage, durch welche funktionellen Gruppen des Kollagens (Carboxyl-, Guanido-, $\varepsilon$-Amino- und Hydroxylgruppen) die kationischen und anionischen Chrom(III)-komplexe gebunden werden und welche von ihnen für die Ausbildung einer unterteilten Querstreifung verantwortlich sind. Die von Gustavson ${ }^{10-12}$ vertretene Auffassung, daß die Bindung der kationischen Chromkomplexe hauptsächlich durch die Carboxylationen

7 R. S. BeAr, Advances Protein Chem. 7, 69 [1952].

8 W. Grassmann, K. Hannig, H. Endres u. A. Riedel, HoppeSeyler's Z. physiol. Chem. 306, 123 [1956].

9 K. Kühn, U. Hofmann u. W. Grassmann, Naturwissenschaften 46, 512 [1959].

10 K. H. Gustavson, J. Amer. Leather Chemists Assoc. 19, 479 [1924].

11 K. H. Gustavson, J. Amer. chem. Soc. 74, 4608 [1952]. geschieht, ist in der Literatur allgemein anerkannt (vgl. dazu l. c. ${ }^{11,13,14}$ ). Nach Bowes und Kenten ${ }^{13}$, Sykes ${ }^{14}$ und Green ${ }^{15}$ beeinflussen aber auch die Amino- und Hydroxylgruppen die Chrombindung, sie sollen jedoch für die Quervernetzung der Protofibrillen keine Rolle spielen.

Über die Bindung der anionischen Chromkomplexe gehen die Meinungen auseinander. Für die Bindung der Sulfitochrom(III)-komplexe hat GUstavson die Hydroxylgruppen, aber auch die Peptidbindungen verantwortlich gemacht ${ }^{12}$, während er für die Oxalatokomplexe eine Bindung an die Aminogruppen des Kollagens annimmt ${ }^{12,16}$. Dagegen halten Shuttleworth ${ }^{17}$ und Sykes ${ }^{14}$ auch bei den anionischen Chromkomplexen eine Bindung an die Carboxylgruppen für wahrscheinlicher.

\section{Ergebnisse}

1. Chromaufnahme, Schrumpfungs punkt und Querstreifung bei Behandlung von Kollagen mit Chrom(III)-salzlösungen verschiedener Zusammensetzung

Zunächst untersuchten wir, unter welchen Bedingungen der Chromgerbung eine unterteilte Querstreifung auftritt. Da reine Chromchlorid- und Chromsulfatlösungen infolge ihrer sauren Reaktion nicht gerbend wirken, müssen sie zunächst mit Natronlauge oder, wie es in der Praxis geschieht, mit Soda "basisch gestellt" werden. Der Gerber spricht bei Zugabe von $1 / 2$ Äquivalent $\mathrm{NaOH}$ oder $\mathrm{Na}_{2} \mathrm{CO}_{3} / \mathrm{Cr}$ von 16-proz., bei Zugabe von 1 Äquivalent $\mathrm{NaOH}$ bzw. $\mathrm{Na}_{2} \mathrm{CO}_{3}$ von 33-proz. basisch gestellten Lösungen, in denen „basische Chrom(III)komplexe“ vorliegen. Infolge der Alkalizugabe treten zunächst Hydroxylgruppen in die Chromkomplexe ein. Anschließend bilden sich mehrkernige Chromkomplexe, welche man für die gerbende Wirkung verantwortlich macht.

Die von uns verwendeten, mit $\mathrm{NaOH}$ versetzten Chromchloridlösungen enthalten reine Hydroxokomplexe, da die Chloranionen eine zu geringe Komplexaktivität besitzen, um bei normalen Konzentra-

12 K. H. Gustavson, J. Soc. Leather Trades' Chemists 39, 2 [1955].

13 J. H. Bowes u. R. H. Kenten, Biochem. J. 44, 142 [1949].

14 R. L. Sykes, J. Soc. Leather Trades' Chemists 39, 56 [1955].

15 R. W. Green, Biochem. J. 54, 187 [1953].

16 K. H. Gustavson, J. Amer. chem. Soc. 48, 2963 [1926].

17 S. G. Shutrleworth, J. Amer. Leather Chemists Assoc. 47, 387 [1952]. 
K. Küнn und E. GeвнаRDt, Chemische und elektronenoptische Untersuchungen über die Reaktion von Chrom(III)-komplexen mit Kollagen (S.23)

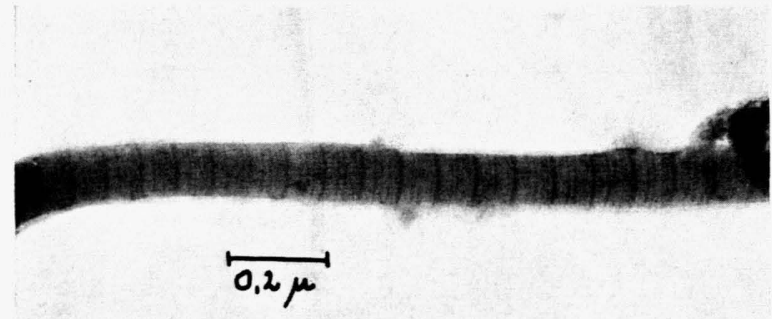

Abb. 1. Kollagenfibrille, behandelt mit Chromchloridlösung (1 Mol NaOH$/ \mathrm{Cr}, 20 \mathrm{mg} \mathrm{Cr} / \mathrm{cm}^{3}$ ) $61 \mathrm{mg} \mathrm{Cr} / \mathrm{g}$ Kollagen, 5- bis 6-fach unterteilte Querstreifung. Nr. 1222/59, elektr.-opt. Vergr. $24100: 1$.

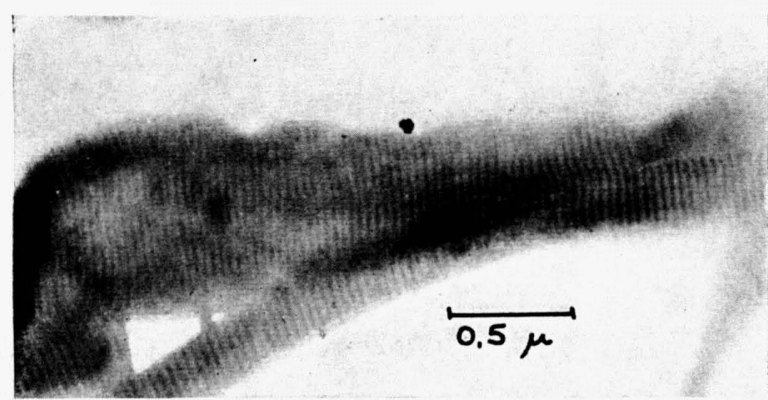

Abb. 3. Kollagenfibrillen, mit Säure gequollen. Keine unterteilte Querstreifung. Nr. 926/57, elektr.-opt. Vergr. 14200 : 1.

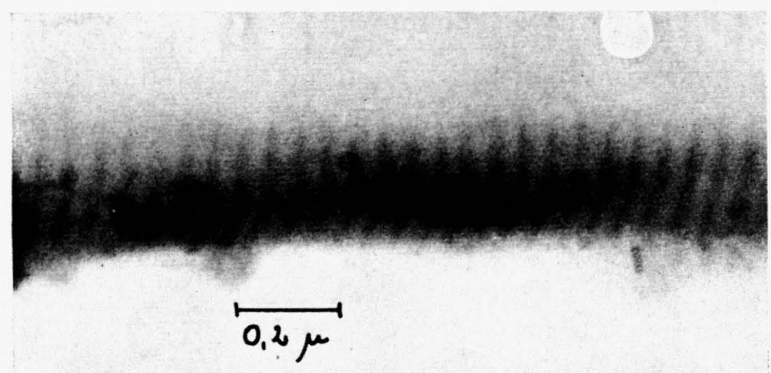

Abb. 5. Methylkollagen, behandelt mit Chromsulfitlösung (6 Äquivalente $\mathrm{Na}_{2} \mathrm{SO}_{3} / \mathrm{Cr}, 1,25 \mathrm{mg} \mathrm{Cr} / \mathrm{cm}^{3}$ ), $56 \mathrm{mg} \mathrm{Cr} / \mathrm{g}$ Kollagen. Keine unterteilte Querstreifung. Nr. 952/59, elektr.-opt. Vergr. 24 $100: 1$

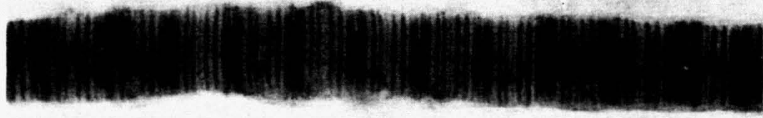

$0.1 \mu$

Abb. 2. Kollagenfibrille, behandelt mit Chromsulfatlösung (1 Mol NaOH/Cr, $5 \mathrm{mg} \mathrm{Cr} / \mathrm{cm}^{3}$ ) $54 \mathrm{mg} \mathrm{Cr} / \mathrm{g}$ Kollagen, 12 -fach unterteilte Querstreifung. Nr. 1126/58, elektr.-opt. Vergr. $50000: 1$.

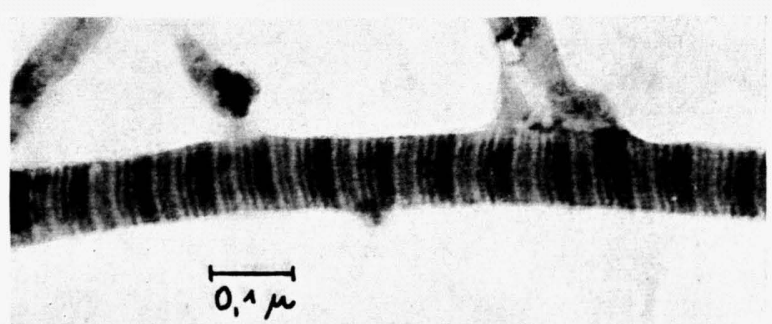

Abb. 4. Kollagenfibrille, mit Säure gequollen, behandelt mit Chromsulfitlösung (6 Äquivalente $\mathrm{Na}_{2} \mathrm{SO}_{3} / \mathrm{Cr}, 1,25 \mathrm{mg} \mathrm{Cr}$ ) $\mathrm{cm}^{3}$ ) $246 \mathrm{mg} \mathrm{Cr} / \mathrm{g}$ Kollagen, 6-7-fach unterteilte Querstreifung. Nr. 900/59, elektr.-opt. Vergr. $24100: 1$.

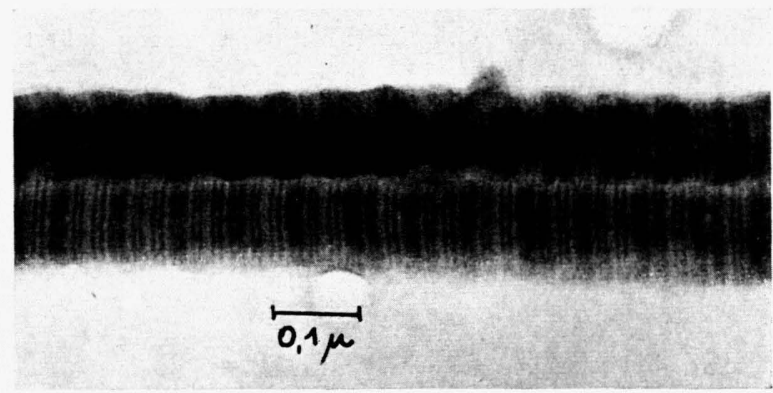

Abb. 6. Desamino-acetylkollagen, behandelt mit Chromsulfitlösung (6 Äquivalente $\mathrm{Na}_{2} \mathrm{SO}_{3} / \mathrm{Cr}, 1,25 \mathrm{mg} \mathrm{Cr} / \mathrm{cm}^{3}$ ), $74 \mathrm{mg}$ $\mathrm{Cr} / \mathrm{g}$ Kollagen, 7-8-fach unterteilte Querstreifung. Nr. 953/59, elektr.-opt. Vergr. $24100: 1$. 
A. KRIEG, Elektronenmikroskopische Untersuchungen zur Rickettsiose von Melolontha melolontha (L.) an Hand von UltraDünnschnitten (S.31)
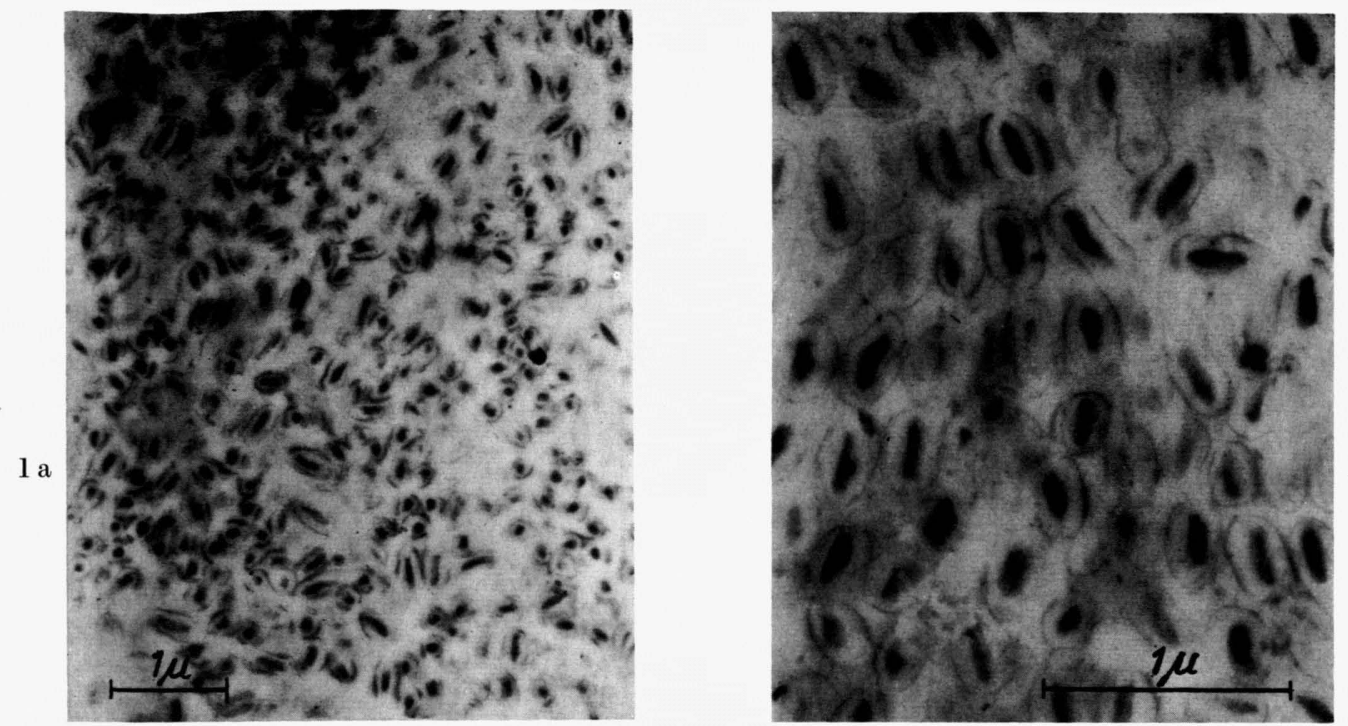

$1 \mathrm{~b}$

Abb. 1. Mit Rickettsiella infizierte Zelle (Spätstadium) (Fixation: saures $\mathrm{OsO}_{4}$; nicht kontrastiert) Abb. M.: a) $12800: 1$; b) $25400: 1$. Völlig von Rickettsiellen erfülltes Cytoplasma.
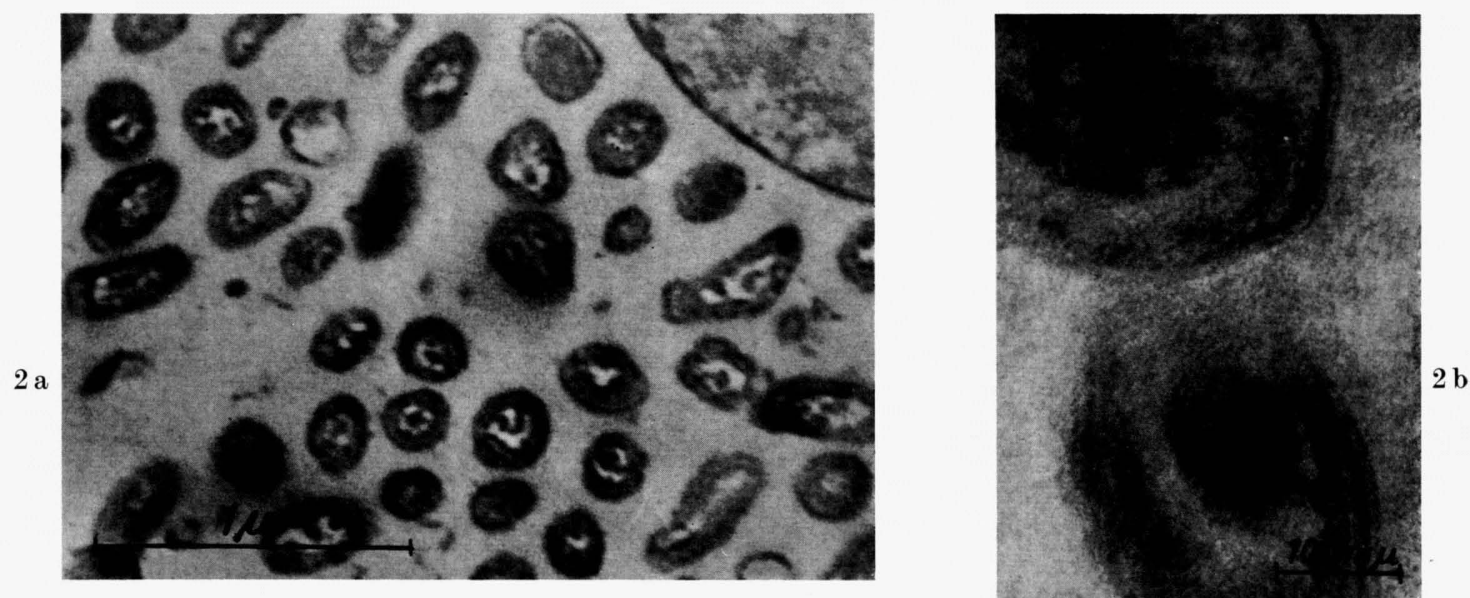

Abb. 2. Rickettsiella melolontha (Fixation: veronal-gepuffertes $\mathrm{OsO}_{4}$; nicht kontrastiert) Abb. M.: a) $32000: 1$; b) $120000: 1$.

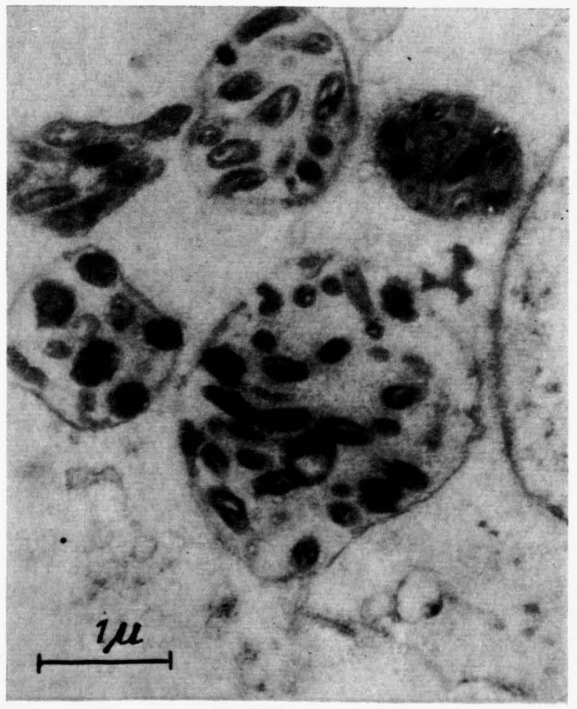

Abb. 3. Mit Rickettsiella infizierte Zelle (Frühstadium) (Fixation: $\mathrm{OsO}_{4}+\mathrm{K}_{2} \mathrm{Cr}_{2} \mathrm{O}_{7}$; nicht kontrastiert) Abb. M.: 12800 : 1. - Initialkörper (homolog den NR-bodies) mit Rickettsien gefüllt.

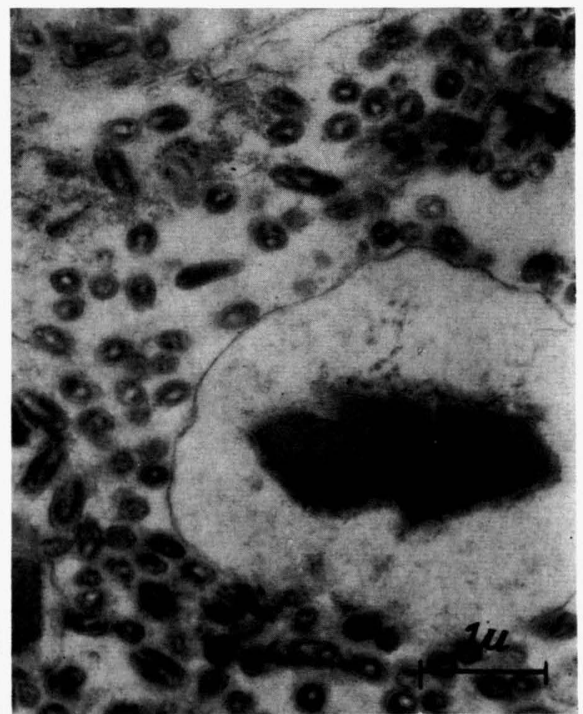

Abb. 4. Mit Rickettsiella infizierte Zelle (Spätstadium) Fixation: $\mathrm{OsO}_{4}+\mathrm{K}_{2} \mathrm{Cr}_{2} \mathrm{O}_{7}$; Kontrastierung: $\mathrm{P}_{2} \mathrm{O}_{5}\left(\mathrm{WO}_{3}\right)_{24}+$ $\mathrm{UO}_{2}\left(\mathrm{C}_{2} \mathrm{H}_{3} \mathrm{O}_{2}\right)$. Abb. M.: $12800: 1-$ Cytoplasma von Rickettsien erfüllt; $\mathrm{A}=$ Albuminoidsphären; $\mathrm{K}=$ Begleitkristalle. 
tionen in die Komplexe mit einzutreten (Formel I) ${ }^{18}$. Bei den mit Soda versetzten Lösungen können auch noch $\mathrm{CO}_{3}{ }^{2 \ominus}$-Anionen in die Komplexe aufgenommen werden.

Die in den basisch gestellten Chromsulfatlösungen vorliegenden Chromkomplexe enthalten neben den Hydroxylgruppen auch noch Sulfatanionen. Formel II gibt den nach KüNTzel und Mitarbb. ${ }^{19}$ formulierten Grundtyp solcher Sulfatochromkomplexe wieder.

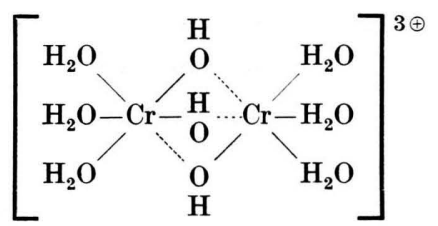

I. Hydroxochrom-Komplex formuliert nach KüNtzeL ${ }^{18}$.<smiles>CCO[C@@H](O)[C@@H](O)OS(=O)(=O)O[C@H](C)O</smiles>

II. Sulfatochrom-Komplex formuliert nach Küntzel u. Mitarbb. ${ }^{19}$.

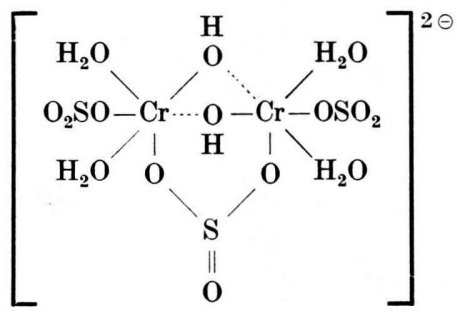

III. Sulfitochrom-Komplex formuliert nach ERDManN 20

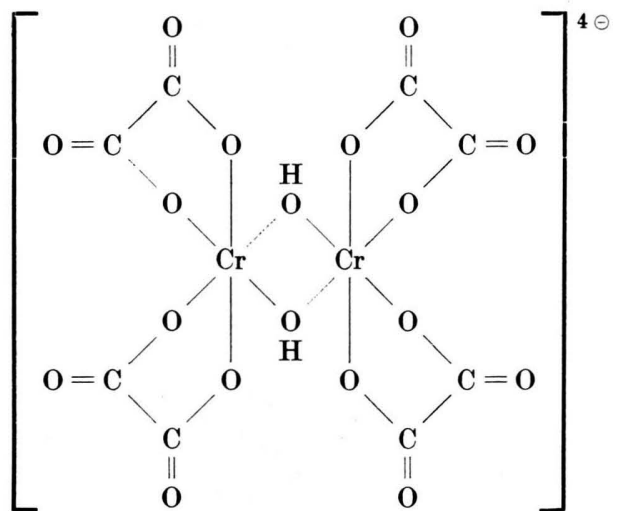

IV. Tetraoxalatodioldichromat (III) nach WERNER ${ }^{21}$. Tab. $1-3$.

Die von uns verwendeten Sulfitochromkomplexe wurden durch Zugabe von 6 Äquivalent $\mathrm{Na}_{2} \mathrm{SO}_{3} / \mathrm{Cr}$ zu Chromchloridlösungen hergestellt. Nach ERDMANN ${ }^{20}$ treten dabei mehrere Sulfitanionen auf Grund ihrer großen Komplexaktivität in den Chromkomplex ein, so daß dieser anionisch wird (Formel III).

Aus demselben Grunde sind auch Oxalatochromkomplexe anionisch. Formel IV gibt das von uns verwendete und nach WERNER ${ }^{21}$ kristallisiert hergestellte Na-tetraoxalatodioldichromat-(III) wieder.

Es ergab sich, daß alle von uns angesetzten Chrom (III) -salzlösungen, bis auf die Lösung des Na-tetraoxalatodioldichromats, eine im Elektronenmikroskop sichtbare, unterteilte Querstreifung erzielten. Unterteilung und Schärfe der Querstreifungsmuster waren aber verschieden (Tab. 1 a und $1 \mathrm{~b}$ ). So zeigten die Perioden von $600-700 \AA$ nach Behandeln mit basischen Chromchlorid- und Chromsulfitlösungen vier bis acht, nach Behandeln mit basischen Chromsulfatlösungen vier bis zwölf Querstreifen (Abb. $1^{*}$ und 2 ).

\begin{tabular}{|c|c|c|c|c|c|c|c|c|c|c|c|c|c|}
\hline \multirow{2}{*}{ Chrombrühe } & \multirow{2}{*}{$\begin{array}{c}\text { Konz. } \\
\text { d. Lösg. } \\
{\left[\mathrm{mg} \mathrm{Cr} / \mathrm{cm}^{3}\right]}\end{array}$} & \multicolumn{3}{|c|}{$\begin{array}{l}1 / 2 \text { Äquiv. } \mathrm{OH}^{-} \\
\text {bzw. } \mathrm{CO}_{3}{ }^{2} \odot / \mathrm{Cr}\end{array}$} & \multicolumn{3}{|c|}{$\begin{array}{l}1 \text { Äquiv. } \mathrm{OH}^{-} \\
\text {bzw. } \mathrm{CO}_{3}{ }^{2} \odot / \mathrm{Cr}\end{array}$} & \multicolumn{3}{|c|}{$\begin{array}{l}1^{1 / 2} \text { Äquiv. } \mathrm{OH}^{-} \\
\text {bzw. } \mathrm{CO}_{3}^{2} \odot / \mathrm{Cr}\end{array}$} & \multicolumn{3}{|c|}{$\begin{array}{l}2 \text { Äquiv. } \mathrm{OH}^{-} \\
\text {bzw. } \mathrm{CO}_{3}{ }^{2} / \mathrm{Cr}\end{array}$} \\
\hline & & $\begin{array}{c}{[\mathrm{mg}} \\
\mathrm{Cr} / \mathrm{g}]\end{array}$ & $T \mathrm{~s}$ & $\mathbf{Q u}$ & $\begin{array}{l}{[\mathrm{mg}} \\
\mathrm{Cr} / \mathrm{g}]\end{array}$ & $T \mathrm{~s}$ & $\mathrm{Qu}$ & $\begin{array}{l}{[\mathrm{mg}} \\
\mathrm{Cr} / \mathrm{g}]\end{array}$ & $T \mathrm{~s}$ & $\mathrm{Qu}$ & $\begin{array}{l}{[\mathrm{mg}} \\
\mathrm{Cr} / \mathrm{g}]\end{array}$ & $T \mathrm{~s}$ & $\mathrm{Qu}$ \\
\hline $\begin{array}{l}\mathrm{Cr} \text {-chlorid }+\mathrm{NaOH} \\
\text { Cr-chlorid }+\mathrm{Na}_{2} \mathrm{CO}_{3}\end{array}$ & $\begin{array}{l}20 \\
20\end{array}$ & $\begin{array}{l}46 \\
14\end{array}$ & $\begin{array}{l}83 \\
78\end{array}$ & $\begin{array}{l}++ \\
++\end{array}$ & $\begin{array}{l}61 \\
18\end{array}$ & $\begin{array}{l}87 \\
85\end{array}$ & $\begin{array}{l}++ \\
++\end{array}$ & $\begin{array}{l}73 \\
25\end{array}$ & $\begin{array}{l}96 \\
90\end{array}$ & $\begin{array}{l}++ \\
++\end{array}$ & $\begin{array}{l}117 \\
34\end{array}$ & $\begin{array}{l}98 \\
96\end{array}$ & $\begin{array}{l}++ \\
++\end{array}$ \\
\hline $\begin{array}{l}\mathrm{Cr} \text {-alaun }+\mathrm{NaOH} \\
\mathrm{Cr} \text {-alaun }+\mathrm{NaOH} \\
\mathrm{Cr} \text {-alaun }+\mathrm{NaOH} \\
\mathrm{Cr} \text {-alaun }+\mathrm{Na}_{2} \mathrm{CO}_{3} \\
\mathrm{Cr} \text {-alaun }+\mathrm{Na}_{2} \mathrm{CO}_{3}\end{array}$ & $\begin{array}{r}20 \\
10 \\
5 \\
20 \\
10\end{array}$ & $\begin{array}{l}33 \\
42 \\
51 \\
31 \\
39\end{array}$ & $\begin{array}{l}90 \\
92 \\
91 \\
77 \\
94\end{array}$ & $\begin{array}{l}++ \\
++ \\
++ \\
++ \\
++\end{array}$ & $\begin{array}{l}48 \\
71 \\
54 \\
34 \\
67\end{array}$ & $\begin{array}{l}97 \\
96 \\
89 \\
81 \\
97\end{array}$ & $\begin{array}{l}+++ \\
+++ \\
+++ \\
++ \\
+++\end{array}$ & $\begin{array}{l}-\overline{89} \\
96 \\
50 \\
92\end{array}$ & $\begin{array}{l}- \\
90 \\
93 \\
96 \\
93\end{array}$ & $\begin{array}{l}-\overline{+} \\
+++ \\
+++ \\
++ \\
+++\end{array}$ & & & \\
\hline
\end{tabular}

Tab. 1 a. Chromaufnahme, Schrumpfungspunkt und Querstreifung von Kollagen bei Behandlung mit kationischen Chrom(III) -komplexen verschiedener Zusammensetzung.

Chromaufnahme in mg Cr/g Kollagen. $T \mathrm{~s}=$ Schrumpfungspunkt, bei unbehandeltem Kollagen ca. $66^{\circ} \mathrm{C}$. Qu $=$ Querstreifung $+++=8-12$,

18 A. Küntzel, Colloquiumsberichte d. Inst. f. Gerbereichem. Darmstadt, Heft 2, 31 [1948].

* Abb. 1-6 s. Tafel S. 24 a.
19 A. Küntzel, H. Erdmann u. H. Spahrkäs, Leder 3, 73 [1952].

20 H. Erdmann, Leder 7, 198 [1956]. 


\begin{tabular}{|l|c|c|c|c|}
\hline \multicolumn{1}{|c|}{ Chrombrühe } & $\begin{array}{c}\text { Konz. d. } \\
\text { Lösung } \\
{[\mathrm{mg}} \\
\left.\mathrm{Cr} / \mathrm{cm}^{3}\right]\end{array}$ & $\begin{array}{c}{[\mathrm{mg}} \\
\mathrm{Cr} / \mathrm{g}]\end{array}$ & Ts & $\mathrm{Qu}$ \\
\hline $\begin{array}{l}\text { Cr-chlorid } \\
+6 \text { Äquiv. }\end{array}$ & 1,25 & 61 & 80 & ++ \\
$\begin{array}{l}\text { Na.SO } \\
\text { Na-tetraoxalatodiol- } \\
\text { dichromat (III) }\end{array}$ & 3,50 & 5 & 68 & + \\
\hline
\end{tabular}

Tab. 1 b. Chromaufnahme, Schrumpfungspunkt und Querstreifung von Kollagen bei Behandlung mit anionischen Chrom (III) -komplexen.

Chromaufnahme in $\mathrm{mg} \mathrm{Cr} / \mathrm{Kollagen}$. $T \mathrm{~s}=$ Schrumpfungspunkt, bei unbehandeltem Kollagen ca. $66^{\circ} \mathrm{C}$. Qu $=$ Querstreifung $++=4-8,+=2-4$ Querstreifen.

Dies stimmt mit den Erfahrungen in der Gerberei überein, wo man ebenfalls mit Chromsulfatgerbbrühen die besten Leder erhält.

Von den in Tab. 1 a angegebenen Gerblösungen mit kationischen Chromkomplexen verwendeten wir $\mathrm{zu}$ den weiteren Gerbversuchen nur noch die mit $\mathrm{NaOH}$ 33-proz. basisch gestellte Chromalaunlösung mit $5 \mathrm{mg} \mathrm{Cr} / \mathrm{cm}^{3}$, da wir mit dieser Lösung die best- unterteilte Querstreifung und die am wenigsten verschmutzten Fibrillen erhielten.

Es ist bemerkenswert, daß die Erhöhung des Schrumpfungspunktes und die Ausbildung einer unterteilten Querstreifung im großen und ganzen parallel miteinander verlaufen. Je höher der Schrumpfungspunkt, desto schärfer unterteilt ist im allgemeinen auch die Querstreifung.

Stöchiometrische Beziehungen zwischen den funktionellen Gruppen im Kollagen ließen sich nicht feststellen. Die Chromaufnahme variiert mit der Zusammensetzung, Konzentration und Basizität der Lösungen.

2. Die Reaktionzwischenkationischen und anionischen Chrom(III)-komplexen und Kollagenpräparaten mit veränderten funktionellen Gruppen

Um festzustellen, welche funktionellen Gruppen des Kollagens für die Ausbildung der unterteilten

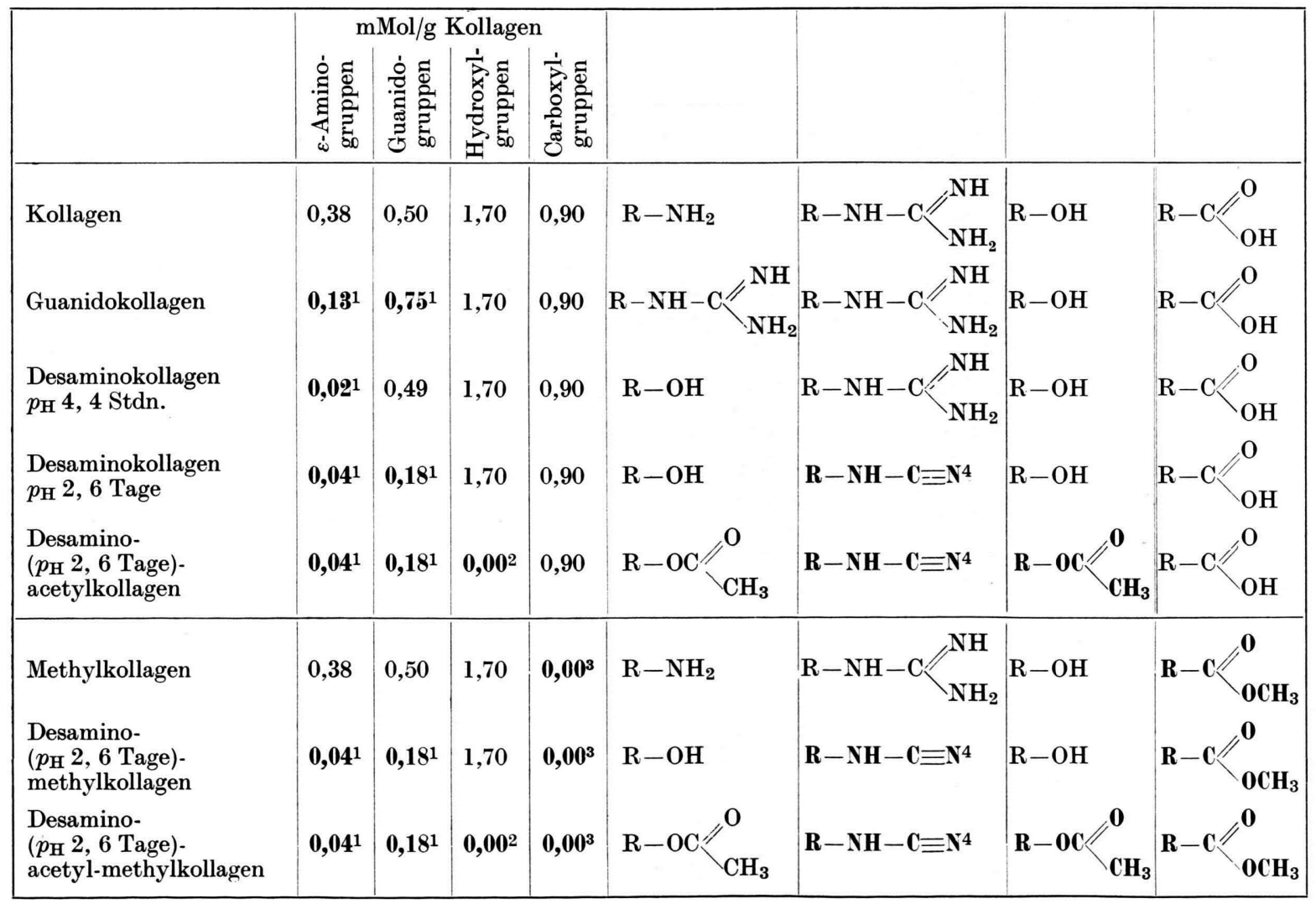

Tab. 2. Veränderung der sauren und basischen Gruppen und der Hydroxylgruppen im Kollagen. 1. Werte von KüHN, Hofmans und Grassmans ${ }^{1}$ übernommen. 2. Acetylgehalt bestimmt nach Bose und JosePH ${ }^{24}$. 3. Methoxylgruppen bestimmt nach Z ZiseL ${ }^{26}$ 4. Formuliert nach Bowes und Kenten ${ }^{13}$. 
Querstreifung nach Chromgerbung verantwortlich sind, veränderten wir systematisch die basischen und sauren Seitenketten des Kollagens (Tab. 2).

Im Guanidokollagen sind die schwach basischen $\varepsilon$-Aminogruppen in die stärker basischen Guanidogruppen umgewandelt, im Desaminokollagen $p_{\mathrm{H}} 4$, 4. Stdn., sind alle $\varepsilon$-Aminogruppen und im Desaminokollagen $p_{\mathrm{H}}$ 2, 6 Tage, auch noch ein großer Teil der Guanidogruppen durch nicht basische Gruppen ersetzt.

Im Acetylkollagen wurden die Hydroxylgruppen blockiert und im Methylkollagen die Carboxylgruppen verestert. So enthält das Desamino-acetylkollagen keine basischen Gruppen und keine Hydroxylgruppen, aber noch die Carboxylgruppen und das Desamino-acetyl-methylkollagen praktisch keine Gruppen mehr, die das Chrom zu binden vermögen.

In der rechten Hälfte der Tab. 2 kann man an Hand von Formelbildern die chemischen Veränderungen der funktionellen Gruppen erkennen.

Zur Gerbung dieser Präparate wurden 1. als kationische Chromkomplexe Sulfatochrom(III)-komplexe, 2. die diesen ähnlich aufgebauten anionischen Sulfitochrom(III)-komplexe und 3. das kristallisiert hergestellte Na-tetraoxalatodioldichromat(III) verwendet.

Der obere Teil der Tab. 3 zeigt, daß bei der Behandlung der Präparate mit Sulfatochrom(III)komplexen die Vermehrung der Guanidogruppen, das Beseitigen der basischen Gruppen und die Blok- kierung der Hydroxylgruppen nur unwesentliche Veränderungen der Schrumpfungspunkte und der unterteilten Querstreifungsmuster zur Folge hatte. Es findet also eine Gerbung statt, auch wenn die Chromaufnahme bis auf die Hälfte vermindert wird. Sobald jedoch die Carboxylgruppen des Kollagens blockiert sind (unterer Teil der Tab. 3), findet keine Gerbung mehr statt. Die durch die Veresterung der Carboxylgruppen bedingte Quellung der Kollagenfibrillen geht durch die Chromgerbung nicht zurück. Die Bestimmung der Schrumpfungspunkte ist daher nicht möglich. Im Elektronenmikroskop erkennt man nur noch eine sehr verschwommene Querstreifung. Die Chromaufnahme nimmt stark ab (Tab. 3).

Die anionischen Sulfitochrom(III)-komplexe verhalten sich ähnlich wie die kationischen Sulfatokomplexe. Alle Kollagenpräparate mit intakten Carboxylgruppen zeigen erhöhte Schrumpfungspunkte und gut unterteilte Querstreifungsmuster. Sind die Carboxylgruppen aber blockiert, tritt keine Gerbung mehr ein (Tab. 3).

Nach Gustavson hängt die Chromaufnahme nach Gerbung mit Sulfitokomplexen sehr von dem Quellungszustand der Kollagenfibrillen $\mathrm{ab}^{12}$. Auch in unseren Versuchen nimmt säuregequollenes Kollagen ein Vielfaches an Chrom auf als natives Kollagen. Obwohl säuregequollenes Kollagen und Methylkollagen ungefähr denselben Quellungszustand aufweisen, bindet das letztere bedeutend weniger

\begin{tabular}{|c|c|c|c|c|c|c|c|c|c|c|c|c|c|c|}
\hline & \multicolumn{2}{|c|}{$\begin{array}{l}\text { unbe- } \\
\text { handelt }\end{array}$} & \multicolumn{3}{|c|}{$\begin{aligned} & \text { Chromalaun } \\
+ & 1 \mathrm{Mol} \mathrm{OH}-/ \mathrm{Cr}\end{aligned}$} & \multicolumn{3}{|c|}{$\begin{array}{l}\text { Chromchlorid } \\
+6 \text { Äquiv. } \\
\mathrm{Na}_{2} \mathrm{SO}_{3} / \mathrm{Cr}\end{array}$} & \multicolumn{6}{|c|}{$\begin{array}{l}\text { Na-tetraoxalatodioldichromat (III) } \\
\qquad \text { m. Säure hydrolys. }\end{array}$} \\
\hline & $T \mathrm{~s}$ & $\mathrm{Qu}$ & $\underset{\mathrm{Cr} / \mathrm{g}]}{[\mathrm{mg}}$ & $T \mathrm{~s}$ & $\mathrm{Qu}$ & $\underset{\mathrm{Cr} / \mathrm{g}]}{[\mathrm{mg}}$ & $|T \mathrm{~s}|$ & $\mathrm{Qu}$ & $\underset{\mathrm{Cr} / \mathrm{g}]}{[\mathrm{mg}}$ & $T \mathrm{~s}$ & $\mathrm{Qu}$ & $\underset{\mathrm{Cr} / \mathrm{g}]}{[\mathrm{mg}}$ & $T \mathrm{~s}$ & $\mathrm{Qu}$ \\
\hline Kollagen & 66 & + & 54 & $|89|$ & +++ & 61 & 80 & ++ & 5 & $|68|$ & + & 43 & 70 & ++ \\
\hline Guanidokollagen & 59 & + & 37 & 80 & +++ & 46 & 100 & ++ & 5 & 57 & + & 40 & 76 & ++ \\
\hline $\begin{array}{l}\text { Desaminokollagen } \\
p_{\mathrm{H}} 4,4 \text { Std. }\end{array}$ & 58 & + & 28 & 96 & +++ & 36 & 78 & ++ & 5 & 58 & + & 43 & 72 & ++ \\
\hline $\begin{array}{l}\text { Desaminokollagen } \\
p_{\mathrm{H}} 2,6 \text { Tage }\end{array}$ & 51 & + & 27 & 83 & ++ & 53 & 80 & ++ & 5 & 56 & + & 48 & 76 & ++ \\
\hline $\begin{array}{l}\text { Desamino ( } p_{\mathrm{H}} 2,6 \text { Tage)- } \\
\text { acetylkollagen }\end{array}$ & 45 & + & 30 & 88 & ++ & 74 & 83 & ++ & & & & 30 & 78 & ++ \\
\hline $\begin{array}{l}\text { mit Säure gequollenes } \\
\text { Kollagen }\end{array}$ & - & $(+)$ & 67 & 100 & ++ & 246 & 100 & ++ & & & & 49 & 98 & ++ \\
\hline Methylkollagen & - & $(+)$ & 9 & - & $(+)$ & 56 & -1 & $(+)$ & & & & 21 & -1 & $(+)$ \\
\hline $\begin{array}{l}\text { Desamino ( } p_{\mathrm{H}} 2,6 \text { Tage)- } \\
\text { methylkollagen }\end{array}$ & - & $(+)$ & 5 & - & $(+)$ & 40 & - & $(+)$ & & & & 9 & - & $(+)$ \\
\hline $\begin{array}{l}\text { Desamino ( } p_{\mathrm{H}} 2,6 \text { Tage)- } \\
\text { acetyl-methylkollagen }\end{array}$ & - & $(+)$ & 2 & - & $(+)$ & 18 & - & $(+)$ & & & & 9 & - & $(+)$ \\
\hline
\end{tabular}

Tab. 3. Einfluß der basischen und sauren Gruppen und der Hydroxylgruppen auf die Chrombindung des Kollagens. Chromgehalt in $\mathrm{mg}$ Chrom/g Kollagen. $T \mathrm{~s}=$ Schrumpfungspunkt, $\mathrm{Qu}=$ Querstreifung $+++=8-12,++=4-8$, 
Chrom. Die Chromaufnahme geht von 246 auf 56 mg Chrom/g Kollagen zurück (Tab. 3).

Behandelt man Kollagen mit dem kristallisiert hergestellten und in Wasser wieder aufgelösten $\mathrm{Na}$ tetraoxalatodioldichromat(III), so wird fast kein Chrom aufgenommen. Nach 4 Wochen ist die Chromaufnahme mit $5 \mathrm{mg}$ Chrom/g Kollagen minimal. Schrumpfungspunkt und Querstreifung unterscheiden sich nicht von unbehandeltem Kollagen. Wird dagegen durch Ansäuern der Lösung die Diolbrücke des Oxalatokomplexes aufgespalten, so daß eine Umbildung des Komplexes möglich wird, stellt man schon nach einigen Tagen eine Chromaufnahme fest. Der umgelagerte Oxalatokomplex verhält sich wie die Sulfato- und Sulfitokomplexe. Sobald die Carboxylgruppen blockiert sind, findet auch bei jenem keine Gerbung mehr statt. Der Einfluß der basischen Gruppen auf die Chromaufnahme ist gering. Auffallend ist die geringe Schrumpfungspunkt-Erhöhung bei der Chromoxalatgerbung (Tab. 3).

Im Elektronenmikroskop erkennt man besonders gut, ob eine Gerbung stattgefunden hat, wie an Hand von den mit Sulfitochrom(III) -komplexen behandelten Kollagenpräparaten gezeigt werden soll. In Abb. 3 sieht man die typisch verschwommene Querstreifung einer mit Säure gequollenen Kollagenfibrille. Nach Behandeln des gequollenen Kollagens mit Sulfitochrom(III)-komplexen erkennt man im Elektronenmikroskop eine unterteilte Querstreifung (Abb. 4). Die Quellung ist durch die quervernetzende, gerbende Wirkung der Chromkomplexe beseitigt. Behandelt man dagegen die ebenfalls gequollenen Fibrillen aus Methylkollagen mit Sulfitochromkomplexen, geht die Quellung nicht zurück (Abb. 5). Eine Quervernetzung ist also auch bei anionischen Chrom(III)-komplexen nur über die Carboxylgruppen möglich. An der-unterteilten Querstreifung der mit Sulfitochromkomplexen behandelten Fibrille aus Desamino-acetylkollagen (Abb. 6) erkennt man sofort, daß auch in Abwesenheit der basischen Gruppen und der Hydroxylgruppen eine Quervernetzung stattgefunden hat.

\section{Diskussion}

Aus dem parallelen Verlauf der Schrumpfungspunkt-Erhöhung und der Ausbildung der unterteilten Querstreifung nach Behandeln mit Chromsalzlösungen geht hervor, daß die Gerbwirkung und die Entstehung einer unterteilten Querstreifung auf dieselben Ursachen zurückzuführen sind. Eine festere
Quervernetzung durch die Chromkomplexe hat auch eine bessere Ordnung der aus den Polypeptidspiralen gebildeten Protofibrillen zur Folge.

Ein weiteres Ergebnis dieser Untersuchungen ist der Nachweis, daß die für diese verfestigende und ordnende Quervernetzung der Protofibrillen verantwortliche Bindung der kationischen und anionischen Chromkomplexe ausschließlich über die Carboxylgruppen des Kollagens erfolgt. Die Reaktion ist insoweit also unabhängig von der Ladung der Komplexe ${ }^{14,17}$. Die basischen Gruppen des Kollagens sind für die Quervernetzung der Protofibrillen entbehrlich.

Eine weitere Stütze für die Annahme einer koordinativen Bindung der Chromkomplexe an die Carboxylgruppen des Kollagens ist der Befund, daß nur solche Komplexe mit dem Kollagen quervernetzend reagieren können, die koordinative Stellen für die Aufnahme von mindestens zwei Carboxylgruppen aus benachbarten Protofibrillen frei haben, was der Fall ist, wenn die Komplexe über locker gebundene Wassermoleküle verfügen. Diese Voraussetzung ist bei den Hydroxo-, Sulfato- und Sulfitochrom(III)-komplexen gegeben. An den nach KüNTzel und Mitarbb. ${ }^{19}$ und Erdmann ${ }^{20}$ formulierten Grundtypen dieser Komplexe erkennt man einige nur mit $\mathrm{H}_{2} \mathrm{O}$ besetzte Koordinationsstellen, die leicht durch Carboxylationen des Kollagens besetzt werden können (Formel II und III).

Anders ist es bei dem Tetraoxalatodioldichromat(III)-anion ${ }^{21}$. Hier sind sämtliche Koordinationsstellen durch stark komplexaktive Oxalationen und Hydroxylgruppen besetzt (Formel IV). Die Carboxylationen können nicht in den Komplex eintreten, und es findet trotz der negativen Ladung des Komplexes fast keine Reaktion mit den basischen Gruppen des Kollagens statt (vgl. dagegen l.c. ${ }^{12}$ ). Erst wenn durch Säure eine Hydrolyse der Diolbrücken und damit eine Umlagerung des Komplexes, bei der wahrscheinlich $\mathrm{H}_{2} \mathrm{O}$-Moleküle aufgenommen werden, möglich wird, tritt eine Gerbung ein.

Die durch Chromgerbung bewirkte Erhöhung der Schrumpfungspunkte ist bei ein und demselben Präparat am größten bei Sulfatokomplexen, ein wenig geringer bei Sulfito- und am geringsten bei Oxalatokomplexen. Die Ausbildung der unterteilten Querstreifung zeigt einen ähnlichen Gang. Dies deutet darauf hin, daß die Gegenwart der Sulfito- und vor allem der Oxalatoliganden den Eintritt der Carboxylgruppen in den Chromkomplex erschwert. Aus 
dem gleichen Grunde steigt die Reaktionszeit der Chromkomplexe mit Kollagen in der Reihenfolge Sulfato-, Sulfito- und Oxalatokomplex (s. experimenteller Teil).

Auf welche Art die basischen Gruppen an der Chromaufnahme beteiligt sind, läßt sich aus unseren Versuchen nicht erkennen. Da nach SHUtrLeworth ${ }^{22}$ die Aminogruppen bei den $p_{\mathrm{H}}$-Bedingungen der Chromgerbung nicht koordinativ gebunden werden können, nimmt Sykes ${ }^{23}$ an, daß sie indirekt an der Chrombindung beteiligt sind. Die Aminogruppen sollen durch Übernahme eines Protons die Dissoziation der Carboxylgruppen fördern und so mehr Carboxylationen zur Bindung des Chroms freimachen.

Die Reaktion zwischen Kollagen und Chrom(III) komplexen wird dadurch unübersichtlich, daß sich keine stöchiometrischen Beziehungen zwischen der Chromaufnahme und den Carboxylgruppen des Kollagens finden lassen. Dies hat mehrere Gründe: 1. liegen in den Chromlösungen Gemische verschieden großer und verschieden zusammengesetzter Chromkomplexe vor, die unterschiedliche Affinität zum Kollagen besitzen, 2. sind bei dem $p_{\mathrm{H}}$-Wert $\left(p_{\mathrm{H}} 3-p_{\mathrm{H}} 5\right)$ der Chromgerbung nicht alle Carboxylgruppen dissoziiert, so daß nur ein Teil von ihnen an der Chrombindung teilnehmen kann, 3 . kann die Aufnahme der Chromkomplexe neben der quervernetzenden Bindung an die Carboxylationen vielleicht auch durch andere, keine Gerbung bewirkende Bindungen, z. B. durch WasserstoffbrückenBindungen und van der Wa alsche Kräfte, erfolgen.

Ein Beispiel dafür ist die starke Erhöhung der Chromaufnahme bei Gerbung des säuregequollenen Kollagens mit Sulfitochromkomplexen. So kann man auch verstehen, warum SchrumpfungspunktErhöhung und Ausbildung einer unterteilten Querstreifung nicht immer parallel mit der Chromaufnahme verlaufen.

Abschließend können die Ergebnisse dieser Arbeit folgendermaßen zusammengefaßt werden: Bei der Reaktion von Chrom(III)-komplexen mit Kollagen erfolgt die Gerbung durch eine koordinative, verfestigende Quervernetzung der Protofibrillen über

21 A. Werner, Liebigs Ann. Chem. 406, 261 [1914].

22 S. G. Shuttleworth, J. Soc. Leather Trades' Chemists 34, 410 [1950].

23 R. L. Syкes, J. Soc. Leather Trades' Chemists 38, 51 [1954]. die Carboxylgruppen der Asparagin- und Glutaminsäure. Diese verfestigende Quervernetzung verursacht gleichzeitig eine Ordnung der Protofibrillen im Sinne eines „In-Register-bringens“ der Anhäufungen der polaren und schweren Aminosäuren und damit die Ausbildung einer im Elektronenmikroskop sichtbaren unterteilten Querstreifung.

\section{Experimenteller Teil M a terial}

Als Ausgangsmaterial diente Achillessehne vom Rind. Die Sehnen wurden mit dem Gefriermikrotom geschnitten und anschließend in einem Homogenisator fein verteilt. Die Kollagenfibrillen bleiben bei dieser Präparation nativ und sind so, im Gegensatz zu Hautpulver, zu elektronenmikroskopischen Untersuchungen geeignet. Gegenüber kompakten Hautstückchen erreicht man eine schnellere Durchreaktion und vermeidet eine Einlagerung von vielkernigen Chromkomplexen in die $Z$ wischenräume des Fasergeflechtes, so daß die Chromanalysen nicht so stark verfälscht werden.

$$
\begin{gathered}
\text { Veränderung der funktionellen } \\
\text { Gruppen des Kollagens }
\end{gathered}
$$

Die in Tab. 2 wiedergegebenen Kollagenpräparate mit veränderten sauren und basischen Seitenketten wurden wie folgt hergestellt:

Guanidokollagen, Desaminokollagen ( $p_{\mathrm{H}} 4,4$ Stdn.) und Desaminokollagen ( $p_{\mathrm{H}} 2,6$ Tage) wurden nach der von Kühn, Grassmann und Hofmann angegebenen Methode erhalten ${ }^{1}$.

Desamino( $p_{\mathrm{H}} 2,6$ Tage)-acetylkollagen: Nach einer Vorschrift von Bose und Joseph ${ }^{24}$ wurden $5 \mathrm{~g}$ Desaminokollagen in $50 \mathrm{ml}$ Äthylacetat aufgeschlämmt, mit $30 \mathrm{ml}$ 90-proz. Ameisensäure (als Katalysator) versetzt und $30 \mathrm{ml}$ Essigsäureanhydrid zugegeben. Der E r l e n m e y e $r$ - Kolben wurde gut verschlossen und unter wiederholtem Umschütteln 7 Tage stehen gelassen. Danach wurde das Präparat gut gewaschen und mit Aceton getrocknet.

Methylkollagen: Einer Vorschrift von Burton und Mitarbb. entsprechend ${ }^{25}$, wurde etwa 1 g Kollagen nach vorheriger Trocknung mit Methanol in eine etwa 0,2-n. methanolische Salzsäure gebracht und im verschlossenen Kolben unter öfterem Umschütteln stehen gelassen. Nach 6 Tagen wurde mit Methanol und Äther gewaschen und über $\mathrm{P}_{2} \mathrm{O}_{5}$ und $\mathrm{KOH}$ (zur Bindung der $\mathrm{HCl}$ ) getrocknet.

Desamino( $p_{\mathrm{H}} 2,6$ Tage)-methylkollagen: In der oben beschriebenen Weise wurde auch Desaminokollagen methyliert.

Desamino( $p_{\mathrm{H}} 2,6$ Tage)-methyl-acetylkollagen: Des-

24 S. M. Bose U. K. T. Joseph, Arch. Biochem. Biophysics 74, 46 [1958].

25 D. Burton, J. P. Danby u. R. L. Sykes, J. Soc. Leather Trades' Chemists 37, 219 [1953].

26 L. Gattermann, Praxis d. org. Chemikers, 32. Aufl., 1947, S. 76. 
amino-methylkollagen wurde, wie bei Desamino-acetylkollagen beschrieben, in Äthylacetat mit Essigsäureanhydrid behandelt.

$$
\begin{gathered}
\text { Analyseder chemisch veränderten } \\
\text { Kollagenpräparate }
\end{gathered}
$$

Die Analysenwerte für Guanidokollagen und Desaminokollagen wurden von KüHN, Grassmann und HofMANN ${ }^{1}$ übernommen.

Zur Bestimmung des Acetylgehaltes der acetylierten Kollagenpräparate wurde nach der von Bose und JoSEPH ${ }^{24}$ benutzten Umesterungsmethode verfahren:

$0,5 \mathrm{~g}$ Acetylkollagen wurden in einem mit $35 \mathrm{ml}$ wasserfreiem Methanol beschickten Reaktionskolben gebracht. Nach Zugabe einer 0,2-n. Lösung von Na-methylat wurde $2 \mathrm{Stdn}$. am Rückfluß erhitzt, sodann $25 \mathrm{ml}$ 2- $n . \mathrm{H}_{2} \mathrm{SO}_{4}$ in Methanol zugegeben, überdestilliert und in einer abgemessenen Menge $n / 10-\mathrm{NaOH}$ aufgefangen. Nach dem Überdestillieren von weiteren $40 \mathrm{ml}$ Methanol wurde $1 / 2$ Stde. am Wasserbad erhitzt und nach dem Abkühlen die überschüssige $\mathrm{NaOH}$ mit $\mathrm{HCl}$ gegen $\mathrm{Phe}$ nolphthalein zurücktitriert. Auf die gleiche Weise wurde eine Blindprobe mit normalem Kollagen durchgeführt und der Blindwert vom gefundenen Acetylgehalt abgezogen.

Die quantitative Bestimmung der Methoxylgruppen in den methylierten Kollagenpräparaten wurde nach der Methode von ZeIsel durchgeführt ${ }^{26}$.

Zur Gerbung wurden folgende Chromsalzlösungen verwendet:

1. Chromchloridlösungen $\left(\left[\mathrm{CrCl}_{2}\left(\mathrm{H}_{2} \mathrm{O}\right)_{4}\right] \mathrm{Cl}+2 \mathrm{H}_{2} \mathrm{O}\right)$, Chromgehalt $20 \mathrm{mg} \mathrm{Cr} / \mathrm{cm}^{3} \mathrm{H}_{2} \mathrm{O}$, mit $1 / 2,1,1^{1 / 2}$ und 2 Äquivalenten $\mathrm{NaOH}$ bzw. $\mathrm{Na}_{2} \mathrm{CO}_{3} / \mathrm{Cr}$ versetzt $(16,33$, 50 und $66 \%$ basisch), (Hydroxochromkomplexe).

2. Chromalaunlösungen $\left(\mathrm{KCr}\left(\mathrm{SO}_{4}\right)_{2} \cdot 12 \mathrm{H}_{2} \mathrm{O}\right)$, Chromgehalt 5,10 und $20 \mathrm{mg} \mathrm{Cr} / \mathrm{cm}^{3} \mathrm{H}_{2} \mathrm{O}$, mit $1 / 2,1$ und $1^{1 / 2}$ Äquivalenten $\mathrm{NaOH}$ bzw. $\mathrm{Na}_{2} \mathrm{CO}_{3} / \mathrm{Cr}$ versetzt (Sul. fatochromkomplexe).

3. Chromchloridlösung $\left(\left[\mathrm{CrCl}_{2}\left(\mathrm{H}_{2} \mathrm{O}\right)_{4}\right] \mathrm{Cl}+2 \mathrm{H}_{2} \mathrm{O}\right)$ mit 6 Äquivalenten $\mathrm{Na}_{2} \mathrm{SO}_{3} / \mathrm{Cr}$ versetzt, Chromgehalt $1,25 \mathrm{mg} \quad \mathrm{Cr} / \mathrm{cm}^{3} \quad \mathrm{H}_{2} \mathrm{O}$ (Sulfitochromkomplexe). $\mathrm{Um}$ während der Gerbung eine Oxydation der Sulfitanionen zu vermeiden, wurde unter Stickstoff gearbeitet ${ }^{20}$.

4. Wäßrige Lösung des nach $W_{\text {ERNER }}{ }^{21}$ kristallisiert hergestellten Na-tetraoxalatodioldichromats-(III), Chromgehalt $3,5 \mathrm{mg} \quad \mathrm{Cr} / \mathrm{cm}^{3} \quad \mathrm{H}_{2} \mathrm{O}$ (Oxalatochromkomplex). Zur Untersuchung wurde die Lösung geteilt. Teil A wurde unverändert verwendet $\left(p_{\mathrm{H}} 5,8\right)$, Teil B wurde auf $p_{\mathrm{H}} 3$ angesäuert und stehen gelassen, bis der $p_{\mathrm{H}}$-Wert konstant blieb (nach 3 Tagen $\left.p_{\mathrm{H}} 5,2\right)$.

Der Eintritt der Hydroxylgruppen (Verolung) und die Bildung der mehrkernigen Chromkomplexe geht

27 Soc. Leather Trades' Chemists, Official Methods of Analysis, 1957, S. 86. nur langsam vor sich. Um stets mit gleichartig zusammengesetzten Chromlösungen arbeiten zu können, wurden von jeder Lösung größere Mengen angesetzt, die ca. 3 Monate stehen gelassen wurden (Alterung). Erst dann wurden sie zur Gerbung verwendet.

Die Gerbdauer betrug bei Hydroxo- und Sulfatokomplexen 24 Stdn., bei Sulfitokomplexen $48 \mathrm{Stdn}$. und bei Oxalatkomplexen 4 Wochen (Teil A) bzw. 14 Tage (Teil B).

\section{C h r o m best i m m ung}

Es wurde nach der Methode der Society of Leather Trades Chemists (SLTC) gearbeitet ${ }^{27}$, die wir in den Halbmikromaßstab übertrugen: Etwa $30 \mathrm{mg}$ des mit Chrom behandelten Kollagens wurden mit $1 \mathrm{~cm}^{3}$ rauchender $\mathrm{HNO}_{3}$ und $2 \mathrm{~cm}^{3}$ eines Gemisches von $\mathrm{HClO}_{4}(60 \%)$ und $\mathrm{H}_{2} \mathrm{SO}_{4}(98 \%)$ (3:1) versetzt und bis zur vollständigen Oxydation des $\operatorname{Cr}(\mathrm{III})$ erhitzt. Nach raschem Abkühlen wurden $5 \mathrm{~cm}^{3} 50$-proz. $\mathrm{H}_{2} \mathrm{SO}_{4}$ zugegeben und zur Entfernung des Chlors 10 Min. gekocht. Nach dem Abkühlen wurde gegen $n / 100$-Eisen(II)-ammonsulfatlösung und Ferroin als Indikator titriert. Der Chromgehalt wurde in $\mathrm{mg} \mathrm{Cr} / \mathrm{g}$ Kollagen angegeben.

Die Menge des Kollagens wurde aus dem Stickstoffgehalt (nach $\mathrm{K}$ jeld a hl) der chromgegerbten Präparate berechnet, nachdem vorher die Stickstoffgehalte der mit Alkohol und Äther gewaschenen und über $\mathrm{P}_{2} \mathrm{O}_{5}$ getrockneten ungegerbten Kollagenpräparate bestimmt worden waren.

Die Schrumpfungspunkte wurden ähnlich der Methode von Borasky und Nutring ${ }^{28}$ bestimmt: Die mit Aufschlämmungen von Kollagenfäserchen beschickten Schmelzpunktröhrchen wurden in eine mit $\mathrm{H}_{2} \mathrm{SO}_{4}$ gefüllte Schmelzpunktapparatur gebracht und das Verhalten der Fäserchen beim langsamen Erhitzen durch ein Stereomikroskop betrachtet. Als Schrumpfungstemperatur $\left(T_{\mathrm{s}}\right)$ wurde der Wert angegeben, bei dem sich die Hauptmenge der Fäserchen zu einer Masse zusammengeballt hat. Die Schrumpfungspunkte lagen durchschnittlich um 5-10\% der Celsiusgrade tiefer als im Faserverband.

Zur elektronenmikroskopischen Untersuchung wurden die Fasern in Wasser aufgeschlämmt und auf mit Kollodium befilmte und mit Kohle bedampfte Blenden aufgebracht. Die Untersuchungen wurden mit einem Siemens ÜM $100 \mathrm{e}$ und bei einer Strahlspannung von $80 \mathrm{kV}$ durchgeführt.

Der Deutschen Forschungsgemeinschaft danken wir für großzügige Unterstützung.

Besonderen Dank schulden wir Herrn Prof. Dr. U. HoFmans für wertvolle Anregungen und Diskussionen sowie für die Unterstützung mit den Mitteln des Institutes.

28 R. Borasky u. G. C. Nutring, J. Amer. Leather Chemists Assoc., 44, 830 [1949]. 